\title{
Optimization of conditions for the reaction between $\beta$-lactam carbenes and 3,6-di-(2-thienyl)tetrazine
}

\author{
Xiuqing Yao and Xiaorong Wang* \\ College of Chemistry, Chemical Engineering and Environmental Engineering, \\ Liaoning Shihua University, Fushun, Liaoning 113001, China \\ E-mail:kongquegege@126.com
}

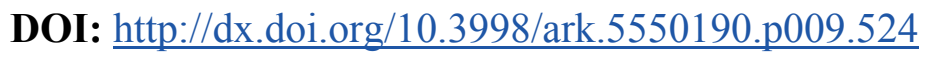

\begin{abstract}
The reaction between $\beta$-lactam carbenes and 3,6-di-(2-thienyl)tetrazine was studied, affording novel 5,8-di(thien-2-yl)-1,6,7-triazaspiro[3.4]octa-5,7-dien-2-ones and 7-(thien-2-yl)-3,3adihydrothieno $\left[2^{\prime}, 3^{\prime}: 3,4\right]$ cyclopenta[1,2-b]pyrrol-2(1H)-ones on heating at approximately $100{ }^{\circ} \mathrm{C}$. The reaction proceeded by cascade mechanisms, and made this series "reactions rich". This study extends the application of $\beta$-lactam carbenes to the synthesis of complex heterocycles.
\end{abstract}

Keywords: $\beta$-Lactam carbenes, tetrazines, cascade reactions, nucleophilic addition

\section{Introduction}

$\beta$-Lactam carbenes (2-azetidinone-4-ylidenes) have attracted considerable interest, not only on account of their ambiphilic reactivity, but also for their synthetic applications, ${ }^{1-7}$ They can be used for constructing mono- or spiro-cyclic, or fused heterocyclic compounds. Recently, Cheng's group has investigated reactions between $\beta$-lactam carbenes with 3,6-diphenyl-, 3,6-di-(2pyridyl)-, 3,6-di-(4-pyridyl)-, and 3,6-di-(2-pyrimidinyl)-tetrazines, ${ }^{5-7}$ From their study it is evident that the pathways of these reactions are controlled by the structures of the aryl substituents of the tetrazines, and various aryl-fused cyclopenta[b]pyrrol-2-one compounds could readily be obtained in high yield. In particular, attention should be paid to the nucleophilic additions between the $\beta$-lactam carbenes and tetrazines as the substituents on the tetrazines affect the reaction efficiency and mechanism. Thus far, the phenyl group (electron donating) and pyridyl or pyrimidinyl groups (electron withdrawing) have not provided clear evidence. ${ }^{5-7}$ Hence, changing the substituent to a more electron-donating group is desirable. To explore the substituent effects of 3,6-diaryltetrazines on the pathways to and construction methods for the synthesis of tri-(five-membered-ring-fused) heterocycles further, we investigated the reaction between $\beta$-lactam carbenes and 3,6-di-(2-thienyl)tetrazine (3). Interestingly, this reaction formed 
intermediates 5 (5,8-di(thien-2-yl)-1,6,7-triazaspiro[3.4]octa-5,7-dien-2-ones) and the final products 9 (3,3a-dihydrothieno[2',3':3,4]cyclopenta[1,2-b]pyrrol-2(1H)-ones) in moderate yields over different heating times, and evidence was obtained that the electron-rich substituent on the tetrazine slowed down the addition. This study has extended this reaction series and provided a strategy for synthesizing novel heterocyclic pyrrol-2-one compounds.

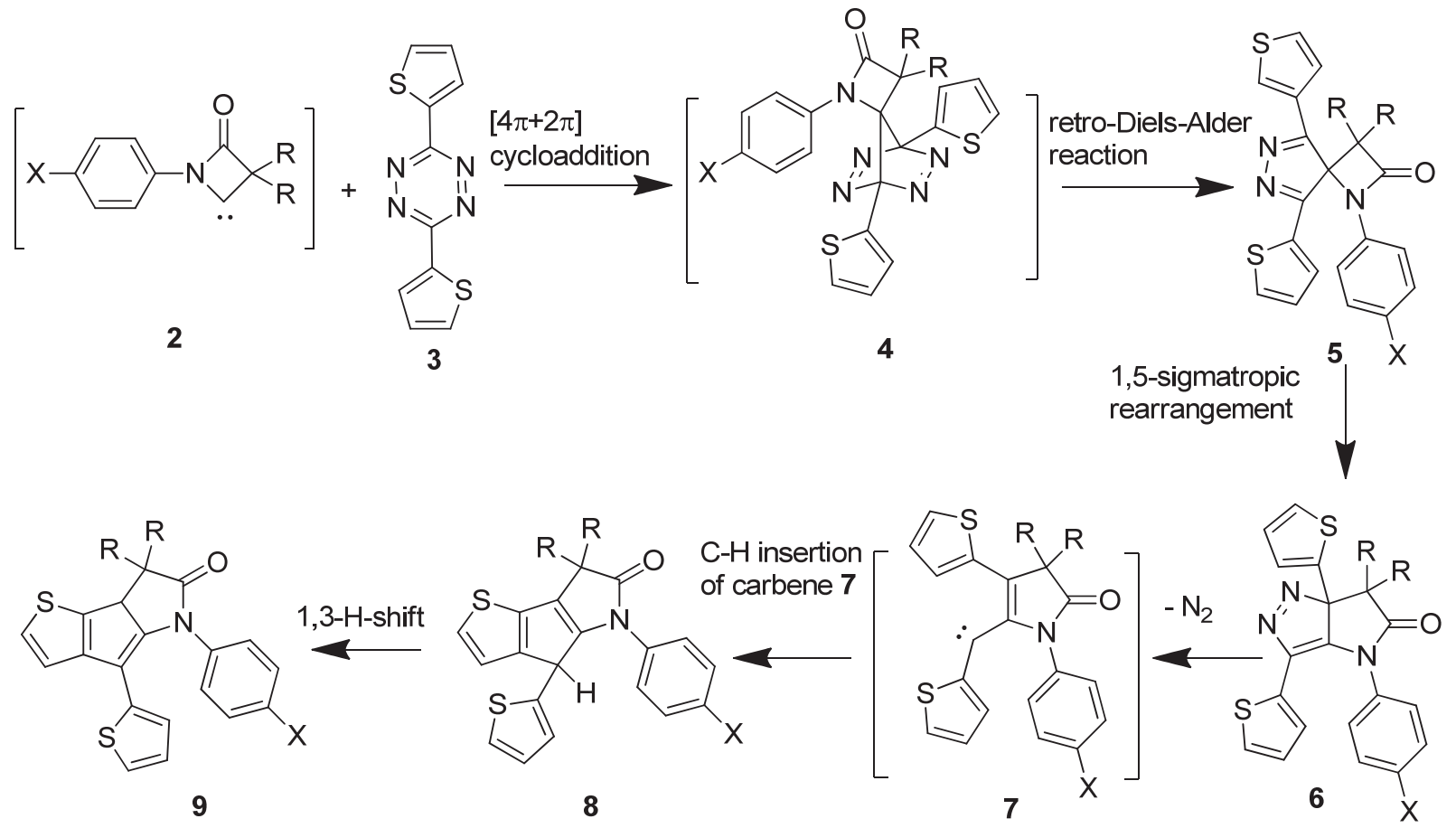

Scheme 1. Proposed mechanisms for the reactions of $\beta$-lactam carbenes with 3,6-di-(2-thienyl)tetrazine.

\section{Results and Discussion}

\section{Synthesis and characterization}

First, the conditions for the reaction between $\beta$-lactam carbenes and 3,6-di-(2-thienyl)tetrazine ${ }^{8}$ were optimized. (Scheme 2) According to previous studies, ${ }^{5-7}$ the optimal temperature for the generation of $\beta$-lactam carbenes $\mathbf{2}$ from spiro-oxadiazolines $\mathbf{1}^{9}$ was in the region of $100-110{ }^{\circ} \mathrm{C}$; thus, several solvents (e.g. toluene, n-heptane, 1,1,2-trichloroethane, 1,4-dioxane) with boiling points in the range of $100-110^{\circ} \mathrm{C}$ were selected; the carbene precursor $\mathbf{1 b}$ and 3,6-di-(2-thienyl)tetrazine (3) in a molar ratio of 1.5:1 were mixed in the solvent and refluxed for $8-24 \mathrm{~h}$ under nitrogen (Table 1). When $\mathbf{1 b}$ was refluxed with $\mathbf{3}$ in toluene, the thin-layer chromatography (TLC) trace indicated the presence of the reaction intermediate as a yellow material (5b). However, the formation of $\mathbf{5 b}$ gradually decreased with reaction time, while product $\mathbf{9 b}$ 
increased, and finally the main product $\mathbf{9 b}$ was obtained in $51 \%$ yield after $16 \mathrm{~h}$, the yields of other byproducts being very low. On the other hand, when the reaction was conducted in $n$-heptane or 1,1,2-trichloroethane the reaction was not satisfactory, with $\mathbf{5 b}$ and $\mathbf{9 b}$ being isolated in low yields (only 10-20\%); When the reaction was conducted under reflux in 1,4dioxane for $8 \mathrm{~h}, \mathbf{5 b}$ and $\mathbf{9 b}$ were isolated in 50\% and 10\% yield respectively; if the reaction time was increased to $24 \mathrm{~h}$, the $\mathbf{5 b}$ changed to $\mathbf{9 b}(59 \%)$, and the yield of $\mathbf{5 b}$ was $\sim 8 \%$; in other words, the intermediate $\mathbf{5 b}$ can be converted into $\mathbf{9 b}$ with a longer heating time. In addition, xylene, with a higher boiling point $\left(140{ }^{\circ} \mathrm{C}\right)$, was used, but $9 \mathrm{~b}$ was obtained in a low yield $(24 \%)$. From these results, it is apparent that this reaction is significantly slower than those reported for the 3,6-diphenyl)-, 3,6-di-(2-pyridyl)-, 3,6-di-(4-pyridyl)-, and 3,6-di-(2-pyrimidinyl)-tetrazines). ${ }^{5-7}$ Compared with the pyridyl or pyrimidinyl substituents, the thienyl group is electron-rich, which decreases the activity of tetrazine toward addition to carbene because the mechanism of this reaction follows nucleophilic addition. According to the results in different solvents (Table 1), dioxane was preferred for this reaction because the total yield was the highest. High temperature (in xylene) resulted in lower yield. We believe that the carbene precursor 1 rapidly decomposes to the carbene at $140{ }^{\circ} \mathrm{C}$; however, carbene 2 did not react with 3 in an efficient manner as the activity of $\mathbf{3}$ is relatively low, resulting in conversion of $\mathbf{2}$ into other substances.

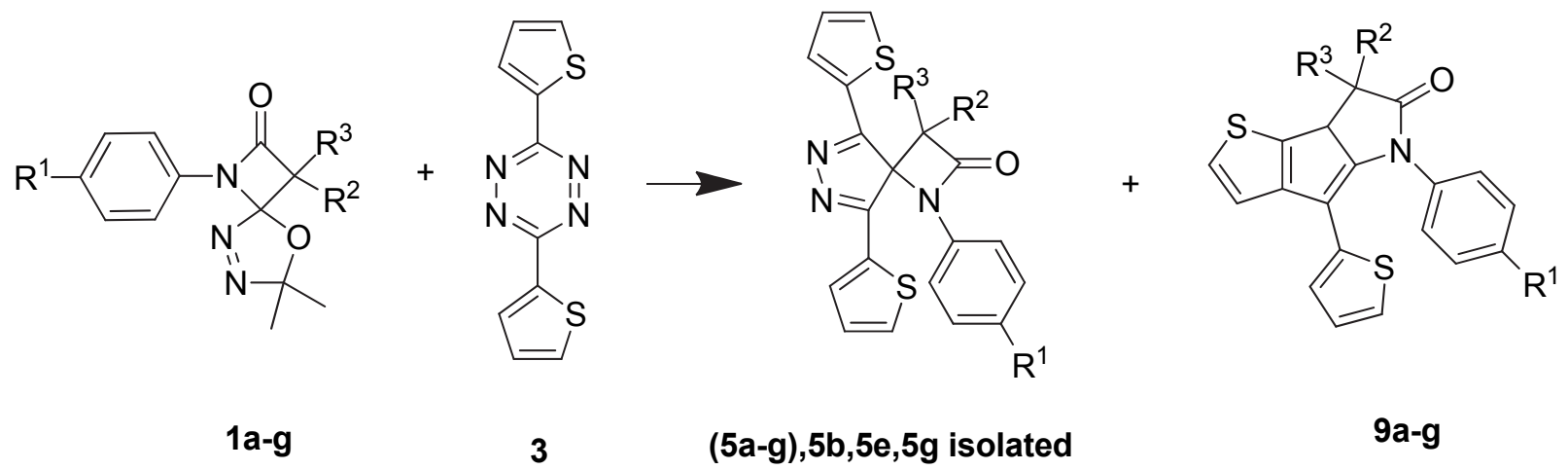

Scheme 2. Effect of different substituents on $\beta$-lactam carbenes precursor 1 and 3,6-di-(2thienyl)tetrazine 3. 1a, 5a, 9a: $\mathrm{R}^{1}=\mathrm{H} ; \mathrm{R}^{2}=\mathrm{R}^{3}=\mathrm{Me}$; 1b, 5b, 9b: $\mathrm{R}^{1}=\mathrm{R}^{2}=\mathrm{R}^{3}=\mathrm{Me}$; 1c, 5c, 9c: $\mathrm{R}^{1}=\mathrm{OCH}_{3} ; \mathrm{R}^{2}=\mathrm{R}^{3}=\mathrm{Me} ; \mathbf{1 d}, \mathbf{5 d}, 9 \mathrm{~d}: \mathrm{R}^{1}=\mathrm{Cl} ; \mathrm{R}^{2}=\mathrm{R}^{3}=\mathrm{Me} ; \mathbf{1 e}, \mathbf{5 e}, 9 \mathrm{e}: \mathrm{R}^{1}=\mathrm{Br} ; \mathrm{R}^{2}=\mathrm{R}^{3}=\mathrm{Me}$; 1f, 5f, 9f: $\mathrm{R}^{1}=\mathrm{Me} ; \mathrm{R}^{2}=\mathrm{R}^{3}=\mathrm{Et} ; \mathbf{1 g}, \mathbf{5 g}, \mathbf{9 g}: \mathrm{R}^{1}=\mathrm{Me} ; \mathrm{R}^{2}, \mathrm{R}^{3}=\left(\mathrm{CH}_{2}\right)_{4}$.

To verify the applicability of all reaction substrates, as indicated in Table 2, carbene precursors 1a-1g bearing different substituents were treated with $\mathbf{3}$, affording $\mathbf{5}$ and $\mathbf{9}$ in moderate yields. When 1a-f and 3 were refluxed in dioxane for 24-26 h, 9 was obtained in 47$65 \%$ yield, while 5 was obtained in approximately 5-18\% yield; when the reaction time was decreased to $7-8 \mathrm{~h}, 5$ was the main product of the reaction (approximately $50 \%$ yield). The amount of carbene precursor $\mathbf{1 g}$ was increased to 2 equivalents as R (cyclopentyl) slowed down the reaction rate. Furthermore, it was difficult to transform intermediate $\mathbf{5 g}$, even if the reaction 
was conducted for $36 \mathrm{~h}$ in refluxing dioxane; therefore, a sequential heating approach was followed, where 3 was first treated with 1 in dioxane, followed by the removal of dioxane, and then xylene was added, which promoted the conversion of $\mathbf{5}$ to afford $\mathbf{9}$; as a result, $\mathbf{5 g}$ was completely converted into $9 \mathrm{~g}$, while $9 \mathbf{d}$ and $9 \mathrm{e}$ were obtained in this manner with slightly improved yields.

Table 1. Optimization of reaction conditions in different solvents $(\mathbf{1 b}: \mathbf{3}=1.5: 1)$

\begin{tabular}{ccccc}
\hline \multirow{2}{*}{ Solvent } & Temperature $\left({ }^{\circ} \mathrm{C}\right)$ & Reaction time $(\mathrm{h})$ & \multicolumn{2}{c}{ Yield (\%) } \\
\cline { 4 - 5 }$n$-heptane & 100 & 12 & $\mathbf{5 b}$ & $\mathbf{9 b}$ \\
\hline 1,1,2-trichloroethane & 110 & 12 & 10 & 8 \\
toluene & 110 & 16 & $-{ }^{a}$ & 51 \\
1,4-dioxane & 100 & 8 & 50 & 10 \\
1,4-dioxane & 100 & 24 & 8 & 59 \\
xylene & 140 & 12 & $-{ }^{a}$ & 24 \\
\hline
\end{tabular}

${ }^{a}$ As these byproducts were obtained in very low yields, they were not isolated.

Table 2. Effect of different substituents on $\beta$-lactam carbene precursors 1 on the reactions between 1 and 3,6-di-(2-thienyl)tetrazine (3) under optimized conditions

\begin{tabular}{|c|c|c|c|c|c|}
\hline \multirow{2}{*}{1} & \multirow{2}{*}{$\mathrm{R}^{1}, \mathrm{R}^{2}, \mathrm{R}^{3}$} & \multirow{2}{*}{$1: 3$} & \multirow{2}{*}{ Reaction conditions } & \multicolumn{2}{|c|}{ Yield $(\%)$} \\
\hline & & & & 5 & 9 \\
\hline $1 \mathbf{a}$ & $\mathrm{H}, \mathrm{CH}_{3}, \mathrm{CH}_{3}$ & $1.5: 1$ & Dioxane, reflux, 24 h & 5a: 5 & 9a: 60 \\
\hline $1 b$ & $\mathrm{CH}_{3}, \mathrm{CH}_{3}, \mathrm{CH}_{3}$ & $1.5: 1$ & Dioxane, reflux, 24 h & 5b: 8 & 9b: 59 \\
\hline $1 \mathrm{c}$ & $\mathrm{OMe}, \mathrm{CH}_{3}, \mathrm{CH}_{3}$ & $1.5: 1$ & Dioxane, reflux, $24 \mathrm{~h}$ & 5c: 6 & 9c: 65 \\
\hline 1d & $\mathrm{Cl}, \mathrm{CH}_{3}, \mathrm{CH}_{3}$ & $1.5: 1$ & Dioxane, reflux, $26 \mathrm{~h}$ & 5d: 14 & 9d: 49 \\
\hline 1e & $\mathrm{Br}, \mathrm{CH}_{3}, \mathrm{CH}_{3}$ & $1.5: 1$ & Dioxane, reflux, $26 \mathrm{~h}$ & 5e: 18 & 9e: 47 \\
\hline 1f & $\mathrm{Me}, \mathrm{Et}, \mathrm{Et}$ & $1.5: 1$ & Dioxane, reflux, $26 \mathrm{~h}$ & $-^{\mathrm{a}}$ & 9f: 60 \\
\hline $1 b$ & $\mathrm{CH}_{3}, \mathrm{CH}_{3}, \mathrm{CH}_{3}$ & $1.5: 1$ & Dioxane, reflux, $8 \mathrm{~h}$ & $\mathbf{5 b}: 50$ & 9b: 10 \\
\hline $1 \mathrm{e}$ & $\mathrm{Br}, \mathrm{CH}_{3}, \mathrm{CH}_{3}$ & $1.5: 1$ & Dioxane, reflux, $7 \mathrm{~h}$ & 5e: 49 & $-^{\mathrm{a}}$ \\
\hline $1 g$ & $\mathrm{Me},\left(\mathrm{CH}_{2}\right)_{4}$ & $2: 1$ & Dioxane, reflux, $36 \mathrm{~h}$ & 5g: 60 & $-{ }^{a}$ \\
\hline $1 g$ & $\mathrm{CH}_{3},\left(\mathrm{CH}_{2}\right)_{4}$ & $2: 1$ & $\begin{array}{l}\text { 1. Dioxane, reflux, } 12 \mathrm{~h} \\
\text { 2. Xylene, reflux, } 6 \mathrm{~h}\end{array}$ & $-{ }^{a}$ & 9g: 51 \\
\hline 1d & $\mathrm{Cl}, \mathrm{CH}_{3}, \mathrm{CH}_{3}$ & $1.5: 1$ & $\begin{array}{l}\text { 1. Dioxane, reflux, } 24 \mathrm{~h} \\
\text { 2. Xylene, reflux, } 3 \mathrm{~h}\end{array}$ & $-{ }^{a}$ & 9d: 54 \\
\hline 1e & $\mathrm{Br}, \mathrm{CH}_{3}, \mathrm{CH}_{3}$ & $1.5: 1$ & $\begin{array}{l}\text { 1. Dioxane, reflux, } 24 \text { h } \\
\text { 2. Xylene, reflux, } 4 \text { h }\end{array}$ & $-{ }^{a}$ & 9e: 57 \\
\hline
\end{tabular}

${ }^{a}$ As these byproducts were obtained in very low yields, they were not isolated. 
The intermediates $\mathbf{5}$ and final products $\mathbf{9}$ were successfully obtained in moderate yields. Scheme 1 shows the mechanism for the reactions between $\beta$-lactam carbene precursor $\mathbf{1}$ and $\mathbf{3}$; first, 5 was obtained by $[4+1]$ cycloaddition, followed by the reverse Diels-Alder reaction, and then it underwent a 1,5-sigmatropic rearrangement, affording $\mathbf{6}$. Intermediate 7 was generated after the removal of a nitrogen molecule, which was followed by intramolecular insertion into a $\mathrm{C}-\mathrm{H}$ bond of the thiophene ring to afford the fused pyrrol-2-ones 8. As the reaction was conducted for a long time under heating conditions, 8 underwent a 1,3-H-shift leading to a double bond rearrangement, affording 9 with a more conjugated, stabler system.

\section{Conclusions}

In summary, we investigated the nucleophilic addition of 2-azetidinon-4-ylidenes (ambiphilic $\beta$ lactam carbenes) with 3,6-di(2-thienyl) tetrazines. Triazaspiro[3.4]octa-5,7-dien-2-ones 5 and pyrrol-2(1H)-ones 9 were obtained as the main products according to the heating time in 1,4dioxane. This study extends the application within $\beta$-lactam carbenes in organic synthesis by providing a synthetic approach to heterocyclic compounds in this series.

\section{Experimental Section}

General. ${ }^{1} \mathrm{H}$ NMR (400 MHz) and ${ }^{13} \mathrm{C}$ NMR (100 MHz) were collected on a Bruker DRX400 spectrometer using the solvent as chemical shift standard. J values are reported in $\mathrm{Hz}$. IR spectra were recorded using an AVATAR 360 FT-IR spectrometer. Mass spectra were recorded on a Surveyor MSQ Plus (ESI) instrument, accurate mass measurement was performed on XEVO G2 QTOF-Waters mass spectrometer and elemental analyses were performed on a GMBH Vario EL instrument. Column chromatography was performed using 200-300 mesh silica gel.

\section{General procedure for the reaction of $\beta$-lactam carbenes with 3,6-di-(2-thienyl)tetrazine}

Method 1 Under nitrogen atmosphere, the mixture of spiro[ $\beta$-lactam-4,2'-oxadiazolines] 1 (1.5 mmol) and 3,6-di-(2-thienyl)tetrazine ${ }^{8} \mathbf{3}$ (1 mmol) was heated in slightly refluxing 1,4dioxane $(50 \mathrm{~mL})$ for $7-8 \mathrm{~h}$ (the time for each reaction was listed in Table 2$)$, After removal of the solvent under vacuum, the residue was purified on a silica gel column eluting with a mixture of petroleum ether and ethyl acetate (50:1-2:1) to give products 5 and $\mathbf{9}$. Products 5 were further purified by recrystallization in ethyl acetate and petroleum ether.

Method 2 Under nitrogen atmosphere, the mixture of spiro[ $\beta$-lactam-4,2'-oxadiazolines] 1 (1.5 $\mathrm{mmol})$ and 3,6-di-(2-thienyl)tetrazine $3(1 \mathrm{mmol})$ was heated in slightly refluxing 1,4-dioxane $(50 \mathrm{~mL})$ for $24-26 \mathrm{~h}$ (the time for each reaction was listed in Table 2), After removal of the solvent under vacuum, the residue was purified on a silica gel column eluting with a mixture of 
petroleum ether and ethyl acetate (50:1-2:1) to give products 5 and $\mathbf{9}$. Products 9 were further purified by recrystallization from ethyl acetate and petroleum ether.

Method 3 Under nitrogen atmosphere, the mixture of spiro[ $\beta$-lactam-4,2'-oxadiazolines] 1 (1.5 $\mathrm{mmol}$ ) and 3,6-di-(2-thienyl)tetrazine 3 (1 mmol) was heated in slightly refluxing 1,4-dioxane $(50 \mathrm{~mL})$ for $12-24 \mathrm{~h}$, After removal of the dioxane under vacuum, xylene $(50 \mathrm{~mL})$ was added for reflux $3-6 \mathrm{~h}$, then the solvent was removed, the residue was purified on a silica gel column eluting with a mixture of petroleum ether and ethyl acetate (50:1-8:1) to give products 9. Products 9 were further purified by recrystallization from ethyl acetate and petroleum ether.

\section{3,3-Dimethyl-1-phenyl-7-(thien-2-yl)-3,3a-dihydrothieno[2',3':3,4]cyclopenta[1,2-b]pyrrol-}

2(1H)-one (9a). Method 2: yield 60\%; mp 161-162 ${ }^{\circ} \mathrm{C}$. IR: $v_{\max }\left(\mathrm{cm}^{-1}\right) 1726,1629 ;{ }^{1} \mathrm{H}$ NMR $\left(400 \mathrm{MHz}, \mathrm{CDCl}_{3}\right) \delta(\mathrm{ppm}) 7.37\left(\mathrm{t},{ }^{3} J_{\mathrm{HH}} 5.0 \mathrm{~Hz}, 1 \mathrm{H}\right), 7.33(\mathrm{~s}, 1 \mathrm{H}), 7.31\left(\mathrm{~d},{ }^{3} J_{\mathrm{HH}} 7.2 \mathrm{~Hz}, 1 \mathrm{H}\right), 7.29$ $\left(\mathrm{d},{ }^{3} J_{\mathrm{HH}} 4.9 \mathrm{~Hz}, 1 \mathrm{H}\right), 7.20\left(\mathrm{t},{ }^{3} J_{\mathrm{HH}} 7.0 \mathrm{~Hz}, 1 \mathrm{H}\right), 7.12\left(\mathrm{~d},{ }^{3} J_{\mathrm{HH}} 5.0 \mathrm{~Hz}, 1 \mathrm{H}\right), 6.74\left(\mathrm{dd},{ }^{3} J_{\mathrm{HH}} 5.0,3.8\right.$ $\mathrm{Hz}, 1 \mathrm{H}), 6.45\left(\mathrm{~d},{ }^{3} J_{\mathrm{HH}} 3.5 \mathrm{~Hz}, 1 \mathrm{H}\right), 3.96(\mathrm{~s}, 1 \mathrm{H}), 1.61(\mathrm{~s}, 3 \mathrm{H}), 0.98(\mathrm{~s}, 3 \mathrm{H}) ;{ }^{13} \mathrm{C} \mathrm{NMR}(100 \mathrm{MHz}$, $\left.\mathrm{CD}_{3} \mathrm{COCD}_{3}\right) \delta(\mathrm{ppm}) 181.6,154.1,146.0,136.9,135.2,133.8,129.5,129.4,127.7,127.4,127.1$, 125.8, 125.0, 121.0, 112.1, 59.5, 47.7, 23.3, 20.8; HRMS (ESI): $364.0831(\mathrm{M}+\mathrm{H})^{+}$; Calcd for $\mathrm{C}_{21} \mathrm{H}_{18} \mathrm{NOS}_{2}$ : 364.0830 .

3,3-Dimethyl-5,8-di(thien-2-yl)-1-(p-tolyl)-1,6,7-triazaspiro[3.4]octa-5,7-dien-2-one

(5b).

Method 1: yield 50\%; mp 145-146 ${ }^{\circ} \mathrm{C}$. IR: $v_{\max }\left(\mathrm{cm}^{-1}\right) 1766,1541,1513 ;{ }^{1} \mathrm{H}$ NMR (400 MHz, $\left.\mathrm{CDCl}_{3}\right) \delta(\mathrm{ppm}) 7.48\left(\mathrm{~d},{ }^{3} J_{\mathrm{HH}} 5.0 \mathrm{~Hz}, 2 \mathrm{H}\right), 7.37\left(\mathrm{~d},{ }^{3} J_{\mathrm{HH}} 3.8 \mathrm{~Hz}, 2 \mathrm{H}\right), 7.09(\mathrm{~s}, 4 \mathrm{H}), 7.03\left(\mathrm{dd},{ }^{3} J_{\mathrm{HH}}\right.$ 4.9, $3.9 \mathrm{~Hz}, 2 \mathrm{H}), 2.26(\mathrm{~s}, 3 \mathrm{H}), 1.32(\mathrm{~s}, 6 \mathrm{H}) ;{ }^{13} \mathrm{C} \mathrm{NMR}\left(100 \mathrm{MHz}, \mathrm{CD}_{3} \mathrm{COCD}_{3}\right) \delta(\mathrm{ppm}) 168.1$, 166.8, 135.7, 134.9, 131.9, 131.3, 131.0, 129.6, 117.4, 82.1, 65.2, 20.8, 20.7; MS (ESI): 406 $(\mathrm{M}+\mathrm{H})^{+}$; Anal. Calcd for $\mathrm{C}_{22} \mathrm{H}_{19} \mathrm{~N}_{3} \mathrm{OS}_{2}$ : C 65.16, H 4.72, N 10.36; Found: C 65.25, H 5.02, N $10.28 \%$.

3,3-Dimethyl-1-(4-methylphenyl)-7-(thien-2-yl)-3,3a-dihydrothieno[2',3':3,4]cyclopenta[1,2-b]pyrrol-2(1H)-one (9b). Method 2: yield 59\%; mp 158-159 ${ }^{\circ} \mathrm{C}$. IR: $v_{\max }\left(\mathrm{cm}^{-1}\right) 1731,1625$, 1510; ${ }^{1} \mathrm{H}$ NMR (400 MHz, $\left.\mathrm{CDCl}_{3}\right) \delta(\mathrm{ppm}) 7.37\left(\mathrm{~d},{ }^{3} J_{\mathrm{HH}} 5.0 \mathrm{~Hz}, 1 \mathrm{H}\right), 7.30\left(\mathrm{~d},{ }^{3} J_{\mathrm{HH}} 5.0 \mathrm{~Hz}, 1 \mathrm{H}\right)$, $7.22\left(\mathrm{~d},{ }^{3} J_{\mathrm{HH}} 8.3 \mathrm{~Hz}, 2 \mathrm{H}\right), 7.13\left(\mathrm{~d},{ }^{3} J_{\mathrm{HH}} 4.0 \mathrm{~Hz}, 1 \mathrm{H}\right), 7.12\left(\mathrm{~d},{ }^{3} J_{\mathrm{HH}} 7.9 \mathrm{~Hz}, 2 \mathrm{H}\right), 6.76\left(\mathrm{dd},{ }^{3} J_{\mathrm{HH}} 5.0\right.$, $3.7 \mathrm{~Hz}, 1 \mathrm{H}), 6.47\left(\mathrm{~d},{ }^{3} J_{\mathrm{HH}} 3.6 \mathrm{~Hz}, 1 \mathrm{H}\right), 3.95(\mathrm{~s}, 1 \mathrm{H}), 2.34(\mathrm{~s}, 3 \mathrm{H}), 1.61(\mathrm{~s}, 3 \mathrm{H}), 0.97(\mathrm{~s}, 3 \mathrm{H}) ;{ }^{13} \mathrm{C}$ NMR (100 MHz, $\left.\mathrm{CDCl}_{3}\right) \delta(\mathrm{ppm}) 181.6,153.3,144.5,136.3,134.4,133.0,132.6,129.4,128.0$, $126.9,126.6,124.6,124.0,120.4,111.6,59.0,47.0,23.3,21.1,20.5 ; \mathrm{MS}(\mathrm{CI}): 378(\mathrm{M}+\mathrm{H})^{+}$; Anal. Calcd for $\mathrm{C}_{22} \mathrm{H}_{19} \mathrm{NOS}_{2}$ : C 69.99, H 5.07 N 3.71; Found: C 69.90, H 5.31, N 3.53\%.

\section{3,3-Dimethyl-1-(4-methoxyphenyl)-7-(thien-2-yl)-3,3a-dihydrothieno[ $\left[2^{\prime}, 3^{\prime}: 3,4\right]$ cyclopenta-}

[1,2-b]pyrrol-2(1H)-one (9c). Method 2: yield 65\%; mp 189-190 ${ }^{\circ} \mathrm{C}$. IR: $v_{\max }\left(\mathrm{cm}^{-1}\right)$ 1720, 1616, 1509; ${ }^{1} \mathrm{H}$ NMR $\left(400 \mathrm{MHz}, \mathrm{CD}_{3} \mathrm{COCD}_{3}\right) \delta(\mathrm{ppm}) 7.55\left(\mathrm{~d},{ }^{3} J_{\mathrm{HH}} 5.0 \mathrm{~Hz}, 1 \mathrm{H}\right), 7.30\left(\mathrm{~d},{ }^{3} J_{\mathrm{HH}} 5.2 \mathrm{~Hz}\right.$, $1 \mathrm{H}), 7.28\left(\mathrm{dd},{ }^{3} J_{\mathrm{HH}} 5.2,0.8 \mathrm{~Hz}, 1 \mathrm{H}\right), 7.26\left(\mathrm{~d},{ }^{3} J_{\mathrm{HH}} 8.8 \mathrm{~Hz}, 2 \mathrm{H}\right), 6.90\left(\mathrm{~d},{ }^{3} J_{\mathrm{HH}} 9.0 \mathrm{~Hz}, 2 \mathrm{H}\right), 6.81$ $\left(\mathrm{dd},{ }^{3} J_{\mathrm{HH}} 5.0,3.7 \mathrm{~Hz}, 1 \mathrm{H}\right), 6.60\left(\mathrm{~d},{ }^{3} J_{\mathrm{HH}} 3.5 \mathrm{~Hz}, 1 \mathrm{H}\right), 4.20(\mathrm{~s}, 1 \mathrm{H}), 3.79(\mathrm{~s}, 3 \mathrm{H}), 1.57(\mathrm{~s}, 3 \mathrm{H}), 0.92$ $(\mathrm{s}, 3 \mathrm{H}) ;{ }^{13} \mathrm{C} \mathrm{NMR}\left(100 \mathrm{MHz}, \mathrm{CD}_{3} \mathrm{COCD}_{3}\right) \delta(\mathrm{ppm}) 180.8,158.3,153.4,145.9,134.4,132.5$, $129.1,128.4,126.7,126.5,125.9,124.8,120.1,113.8,110.4,58.6,54.9,46.5,22.4,20.0$; HRMS (ESI): $394.0942(\mathrm{M}+\mathrm{H})^{+}$; Calcd for $\mathrm{C}_{22} \mathrm{H}_{20} \mathrm{NO}_{2} \mathrm{~S}_{2}: 394.0935$. 
3,3-Dimethyl-1-(4-chlorophenyl)-7-(thien-2-yl)-3,3a-dihydrothieno $\left[2^{\prime}, 3^{\prime}: 3,4\right]$ cyclopenta-

[1,2-b]pyrrol-2(1H)-one (9d). Method 2: yield 54\%; mp 207-208 ${ }^{\circ} \mathrm{C}$. IR: $v_{\max }\left(\mathrm{cm}^{-1}\right)$ 1732, 1621, 1491; ${ }^{1} \mathrm{H}$ NMR $\left(400 \mathrm{MHz}, \mathrm{CD}_{3} \mathrm{COCD}_{3}\right) \delta(\mathrm{ppm}) 7.57\left(\mathrm{~d},{ }^{3} J_{\mathrm{HH}} 4.9 \mathrm{~Hz}, 1 \mathrm{H}\right), 7.39\left(\mathrm{~d},{ }^{3} J_{\mathrm{HH}} 9.0 \mathrm{~Hz}\right.$, 2H), $7.36\left(\mathrm{~d},{ }^{3} J_{\mathrm{HH}} 9.1 \mathrm{~Hz}, 2 \mathrm{H}\right), 7.36(\mathrm{~s}, 1 \mathrm{H}), 7.32\left(\mathrm{~d},{ }^{3} J_{\mathrm{HH}} 5.2 \mathrm{~Hz}, 1 \mathrm{H}\right), 6.87\left(\mathrm{dd},{ }^{3} J_{\mathrm{HH}} 5.0,3.7 \mathrm{~Hz}\right.$, $1 \mathrm{H}), 6.70\left(\mathrm{~d},{ }^{3} J_{\mathrm{HH}} 3.3 \mathrm{~Hz}, 1 \mathrm{H}\right), 4.25(\mathrm{~s}, 1 \mathrm{H}), 1.58(\mathrm{~s}, 3 \mathrm{H}), 0.92(\mathrm{~s}, 3 \mathrm{H}) ;{ }^{13} \mathrm{C} \mathrm{NMR}(100 \mathrm{MHz}$, $\left.\mathrm{CD}_{3} \mathrm{COCD}_{3}\right) \delta(\mathrm{ppm}) 181.6,153.9,145.4,135.7,135.0,134.0,131.7,129.5,129.5,129.5,127.9$, 127.5, 126.2, 121.0, 112.6, 59.3, 47.8, 23.3, 20.7; HRMS (ESI): $398.0449(\mathrm{M}+\mathrm{H})^{+}$; Calcd for $\mathrm{C}_{21} \mathrm{H}_{17} \mathrm{ClNOS}_{2}: 398.0440$.

1-(4-Bromophenyl)-3,3-dimethyl-5,8-di(thien-2-yl)-1,6,7-triazaspiro[3.4]octa-5,7-dien-2-one (5e). Method 1: yield 49\%, mp 165-167 ${ }^{\circ} \mathrm{C}$. IR: $v_{\max }\left(\mathrm{cm}^{-1}\right) 1751,1591,1537 ;{ }^{1} \mathrm{H}$ NMR (400 $\left.\mathrm{MHz}, \mathrm{CD}_{3} \mathrm{COCD}_{3}\right) \delta(\mathrm{ppm}) 7.77\left(\mathrm{dd},{ }^{3} J_{\mathrm{HH}} 5.1,1.0 \mathrm{~Hz}, 2 \mathrm{H}\right), 7.56\left(\mathrm{~d},{ }^{3} J_{\mathrm{HH}} 9.1 \mathrm{~Hz}, 2 \mathrm{H}\right), 7.40(\mathrm{dd}$, $\left.{ }^{3} J_{\mathrm{HH}} 3.8,1.0 \mathrm{~Hz}, 2 \mathrm{H}\right), 7.14\left(\mathrm{dd},{ }^{3} J_{\mathrm{HH}} 5.0,3.8 \mathrm{~Hz}, 2 \mathrm{H}\right), 7.00\left(\mathrm{~d},{ }^{3} J_{\mathrm{HH}} 8.4 \mathrm{~Hz}, 2 \mathrm{H}\right), 1.31(\mathrm{~s}, 6 \mathrm{H}) ;{ }^{13} \mathrm{C}$ NMR (100 MHz, $\left.\mathrm{CD}_{3} \mathrm{COCD}_{3}\right) \delta(\mathrm{ppm}) 167.6,165.5,136.4,133.9,133.1,131.3,130.2,129.0$, 118.6, 117.4, 81.2, 64.8, 19.8; HRMS (ESI): $469.9990(\mathrm{M}+\mathrm{H})^{+}$; Calcd for $\mathrm{C}_{21} \mathrm{H}_{17} \mathrm{BrN}_{3} \mathrm{OS}_{2}$ : 469.9996.

3,3-Dimethyl-1-(4-bromophenyl)-7-(thien-2-yl)-3,3a-dihydrothieno[2',3':3,4]cyclopenta[1,2-b]pyrrol-2(1H)-one (9e). Method 2: yield 57\%; mp 200-201 ${ }^{\circ} \mathrm{C}$. IR: $v_{\max }\left(\mathrm{cm}^{-1}\right)$ 1727, 1617 , 1487; ${ }^{1} \mathrm{H}$ NMR $\left(400 \mathrm{MHz}, \mathrm{CDCl}_{3}\right) \delta(\mathrm{ppm}) 7.42\left(\mathrm{~d},{ }^{3} J_{\mathrm{HH}} 8.4 \mathrm{~Hz}, 2 \mathrm{H}\right), 7.38\left(\mathrm{~d},{ }^{3} J_{\mathrm{HH}} 4.8 \mathrm{~Hz}, 1 \mathrm{H}\right)$, $7.29\left(\mathrm{~d},{ }^{3} J_{\mathrm{HH}} 4.9 \mathrm{~Hz}, 1 \mathrm{H}\right), 7.24\left(\mathrm{~d},{ }^{3} J_{\mathrm{HH}} 8.2 \mathrm{~Hz}, 2 \mathrm{H}\right), 7.19\left(\mathrm{~d},{ }^{3} J_{\mathrm{HH}} 4.8 \mathrm{~Hz}, 1 \mathrm{H}\right), 6.83\left(\mathrm{t},{ }^{3} J_{\mathrm{HH}} 4.0\right.$ $\mathrm{Hz}, 1 \mathrm{H}), 6.55\left(\mathrm{~d},{ }^{3} J_{\mathrm{HH}} 2.9 \mathrm{~Hz}, 1 \mathrm{H}\right), 3.94(\mathrm{~s}, 1 \mathrm{H}), 1.61(\mathrm{~s}, 3 \mathrm{H}), 0.96(\mathrm{~s}, 3 \mathrm{H}) ;{ }^{13} \mathrm{C} \mathrm{NMR}(100 \mathrm{MHz}$, $\left.\mathrm{CD}_{3} \mathrm{COCD}_{3}\right) \delta(\mathrm{ppm}) 181.6,153.9,145.3,136.1,134.5,134.0,132.5,129.6,128.0,127.6,126.5$, 126.3, 121.0, 119.5, 112.7, 59.3, 47.8, 23.3, 20.7; HRMS (ESI): $441.9930(\mathrm{M}+\mathrm{H})^{+}$; Calcd for $\mathrm{C}_{21} \mathrm{H}_{17} \mathrm{BrNOS}_{2}$ : 441.9935 .

3,3-Diethyl-1-(4-methylphenyl)-7-(thien-2-yl)-3,3a-dihydrothieno[2',3':3,4]cyclopenta[1,2-b]pyrrol-2(1H)-one (9f). Method 2: yield 60\%; mp 146-147 ${ }^{\circ} \mathrm{C}$ IR: $v_{\max }\left(\mathrm{cm}^{-1}\right)$ 1719, 1609, 1511; ${ }^{1} \mathrm{H}$ NMR (400 MHz, $\left.\mathrm{CD}_{3} \mathrm{COCD}_{3}\right) \delta(\mathrm{ppm}) 7.55\left(\mathrm{~d},{ }^{3} J_{\mathrm{HH}} 5.0 \mathrm{~Hz}, 1 \mathrm{H}\right), 7.29\left(\mathrm{~d},{ }^{3} J_{\mathrm{HH}} 5.0 \mathrm{~Hz}\right.$, $1 \mathrm{H}), 7.28\left(\mathrm{~d},{ }^{3} J_{\mathrm{HH}} 5.2 \mathrm{~Hz}, 1 \mathrm{H}\right), 7.22\left(\mathrm{~d},{ }^{3} J_{\mathrm{HH}} 8.4 \mathrm{~Hz}, 2 \mathrm{H}\right), 7.15\left(\mathrm{~d},{ }^{3} J_{\mathrm{HH}} 8.3 \mathrm{~Hz}, 2 \mathrm{H}\right), 6.78$ (dd, $\left.{ }^{3} J_{\mathrm{HH}} 5.1,3.7 \mathrm{~Hz}, 1 \mathrm{H}\right), 6.52\left(\mathrm{~d},{ }^{3} J_{\mathrm{HH}} 3.6 \mathrm{~Hz}, 1 \mathrm{H}\right), 4.24(\mathrm{~s}, 1 \mathrm{H}), 2.32(\mathrm{~s}, 3 \mathrm{H}), 2.06-2.12(\mathrm{~m}, 1 \mathrm{H})$, $1.85-1.94(\mathrm{~m}, 1 \mathrm{H}), 1.52-1.62(\mathrm{~m}, 1 \mathrm{H}), 1.28-1.37(\mathrm{~m}, 1 \mathrm{H}), 1.20\left(\mathrm{t},{ }^{3} J_{\mathrm{HH}} 7.5 \mathrm{~Hz}, 3 \mathrm{H}\right), 0.82\left(\mathrm{t},{ }^{3} J_{\mathrm{HH}}\right.$ $7.5 \mathrm{~Hz}, 3 \mathrm{H}) ;{ }^{13} \mathrm{C} \mathrm{NMR}\left(100 \mathrm{MHz}, \mathrm{CD}_{3} \mathrm{COCD}_{3}\right) \delta(\mathrm{ppm}) 180.3,154.2,147.9,137.0,135.4,134.4$, 134.2, 130.0, 129.3, 127.6, 127.4, 125.6, 125.4, 121.2, 110.6, 57.4, 55.2, 27.5, 25.5, 21.0, 9.4, 8.9; HRMS (ESI): $406.1288(\mathrm{M}+\mathrm{H})^{+}$; Calcd for $\mathrm{C}_{24} \mathrm{H}_{24} \mathrm{NOS}_{2}$ : 406.1299.

1-(4-Methylphenyl)-3-(spirocyclopentyl)-5,8-di(thien-2-yl)-1,6,7-triazaspiro[3.4]octa-5,7dien-2-one (5g). Method 1: yield 60\%; mp 166-167 ${ }^{\circ} \mathrm{C}$. IR: $v_{\max }\left(\mathrm{cm}^{-1}\right) 1763,1539,1510 ;{ }^{1} \mathrm{H}$ NMR (400MHz, $\left.\mathrm{CD}_{3} \mathrm{COCD}_{3}\right) \delta(\mathrm{ppm}) 7.78\left(\mathrm{dd},{ }^{3} J_{\mathrm{HH}} 5.1,0.9 \mathrm{~Hz}, 2 \mathrm{H}\right), 7.49\left(\mathrm{dd},{ }^{3} J_{\mathrm{HH}} 4.0,0.9 \mathrm{~Hz}\right.$, 2H), $7.18\left(\mathrm{~d},{ }^{3} v 4.9 \mathrm{~Hz}, 2 \mathrm{H}\right), 7.16\left(\mathrm{~d},{ }^{3} J_{\mathrm{HH}} 5.2 \mathrm{~Hz}, 2 \mathrm{H}\right), 7.05\left(\mathrm{~d},{ }^{3} J_{\mathrm{HH}} 8.5 \mathrm{~Hz}, 2 \mathrm{H}\right), 2.23(\mathrm{~s}, 3 \mathrm{H})$, 2.06-2.09 (m, 2H), 1.53-1.72 (m, 6H); ${ }^{13} \mathrm{C}$ NMR (100MHz, $\left.\mathrm{CD}_{3} \mathrm{COCD}_{3}\right) \delta(\mathrm{ppm}) 169.0,166.7$, 135.6, 134.0, 132.1, 131.2, 130.9, 129.7, 117.3, 81.1, 73.4, 33.1, 25.1, 20.83, 20.79; MS (ESI): $432(\mathrm{M}+\mathrm{H})^{+}$; Anal. Calcd for $\mathrm{C}_{24} \mathrm{H}_{21} \mathrm{~N}_{3} \mathrm{OS}_{2}$ : C 66.79, H 4.90, N 9.74; Found: C 67.01, H 4.88, N $9.66 \%$. 
3-(Spirocyclopentyl)-1-(4-methylphenyl)-7-(thien-2-yl)-3,3a-dihydrothieno[[ $\left.2^{\prime}, 3^{\prime}: 3,4\right]$ cyclopenta[1,2-b]pyrrol-2(1H)-one (9g). Method 3: yield 51\%; mp 126-127 ${ }^{\circ} \mathrm{C}$. IR: $v_{\max }\left(\mathrm{cm}^{-1}\right) 1723$, 1615, 1513; ${ }^{1} \mathrm{H}$ NMR $\left(400 \mathrm{MHz}, \mathrm{CD}_{3} \mathrm{COCD}_{3}\right) \delta(\mathrm{ppm}) 7.57\left(\mathrm{~d},{ }^{3} J_{\mathrm{HH}} 5.0 \mathrm{~Hz}, 1 \mathrm{H}\right), 7.32\left(\mathrm{~d},{ }^{3} J_{\mathrm{HH}} 5.0\right.$ $\mathrm{Hz}, 1 \mathrm{H}), 7.29\left(\mathrm{dd},{ }^{3} J_{\mathrm{HH}} 5.1,0.9 \mathrm{~Hz}, 1 \mathrm{H}\right), 7.24\left(\mathrm{~d},{ }^{3} J_{\mathrm{HH}} 8.4 \mathrm{~Hz}, 2 \mathrm{H}\right), 7.15\left(\mathrm{~d},{ }^{3} J_{\mathrm{HH}} 8.2 \mathrm{~Hz}, 2 \mathrm{H}\right)$, $6.79\left(\mathrm{dd},{ }^{3} J_{\mathrm{HH}} 5.1,3.7 \mathrm{~Hz}, 1 \mathrm{H}\right), 6.56\left(\mathrm{dd},{ }^{3} J_{\mathrm{HH}} 3.6,0.8 \mathrm{~Hz}, 1 \mathrm{H}\right), 4.25(\mathrm{~s}, 1 \mathrm{H}), 2.44-2.52(\mathrm{~m}, 1 \mathrm{H})$, $2.32(\mathrm{~s}, 3 \mathrm{H}), 1.92-2.02(\mathrm{~m}, 3 \mathrm{H}), 1.69-1.74(\mathrm{~m}, 2 \mathrm{H}), 1.56-1.63(\mathrm{~m}, 1 \mathrm{H}), 1.29-1.35(\mathrm{~m}, 1 \mathrm{H}) ;{ }^{13} \mathrm{C}$ NMR $\left(100 \mathrm{MHz}, \mathrm{CD}_{3} \mathrm{COCD}_{3}\right) \delta(\mathrm{ppm}) 181.7,154.6,147.0,136.8,135.3,134.6,134.0,130.0$, $129.4,127.7,127.4,125.7,125.1,121.0,111.2,58.9,58.0,35.9,33.9,27.1,26.3,21.0$; MS (ESI): $404(\mathrm{M}+\mathrm{H})^{+}$; Anal. Calcd for $\mathrm{C}_{24} \mathrm{H}_{21} \mathrm{NOS}_{2}$ : C 71.43, H 5.25, N 3.47; Found: C 71.17, H 5.63, N 3.27\%.

\section{References}

1. Cheng, Y.; Wang, B.; Cheng, L.-Q. J. Org. Chem. 2006, 71, 4418. http://dx.doi.org/10.1021/jo060265h

2. Cheng, Y.; Cheng, L.-Q. J. Org. Chem. 2007, 72, 2625. http://dx.doi.org/10.1021/jo0700631

3. Cheng, L.-Q.; Cheng, Y. Tetrahedron 2007, 63, 9359. http://dx.doi.org/10.1016/j.tet.2007.06.111

4. Shao, N.; Pang, G.-X.; Yan, C.-X.; Shi, G.-F.; Cheng, Y. J. Org. Chem. 2011, 76, 7458. http://dx.doi.org/10.1021/jo201273p

5. Xing, J.; Wang, X.-R.; Yan, C.-X.; Cheng, Y. J. Org. Chem. 2011, 76, 4746. http://dx.doi.org/10.1021/jo200499u

6. Wang, X.-R.; Xing, J.; Yan, C.-X.; Cheng, Y. Org. Biomol. Chem. 2012, 10, 970. http://dx.doi.org/10.1039/C1OB06595E

7. Yan, C.-X.; Zhao, Y.; Wang, X.-R.; Cheng, Y. Tetrahedron 2013, 69, 10279. http://dx.doi.org/10.1016/j.tet.2013.10.034

8. Ye, Q.; Neo, W.-T.; Cho, C.-M.; Yang, S.-W.; Lin, T.; Zhou, H.; Yan, H.; Lu, X. Chi, C.; $\mathrm{Xu}, \mathrm{J}$;; Org. Lett. 2014, 16, 6386. http://dx.doi.org/10.1021/o1503178m

9. Zoghbi, M.; Warkentin, J. J. Org. Chem. 1991, 56, 3214. http://dx.doi.org/10.1021/jo00010a008 